\title{
Seroprevalence of Toxoplasma gondii Infection in Patients of Intensive Care Unit in China: A Hospital Based Study
}

\author{
Yong-Biao Zhang, ${ }^{1}$ Wei Cong, ${ }^{2,3}$ Zhi-Tao Li, ${ }^{1}$ Xiao-Gang Bi, ${ }^{1}$ Ying Xian, ${ }^{1}$ Yan-Hong Wang, \\ Xing-Quan Zhu, ${ }^{2,3,4}$ and Kou-Xing Zhang ${ }^{1}$ \\ ${ }^{1}$ Department of Intensive Care Unit, The Third Affiliated Hospital, Sun Yat-sen University, Guangzhou, Guangdong 510630, China \\ ${ }^{2}$ State Key Laboratory of Veterinary Etiological Biology, Lanzhou Veterinary Research Institute, \\ Chinese Academy of Agricultural Sciences, Lanzhou, Gansu 730046, China \\ ${ }^{3}$ College of Animal Science and Technology, Jilin Agricultural University, Changchun, Jilin 130118, China \\ ${ }^{4}$ Jiangsu Co-Innovation Center for the Prevention and Control of Important Animal Infectious Diseases and Zoonoses, \\ Yangzhou University College of Veterinary Medicine, Yangzhou, Jiangsu 225009, China \\ Correspondence should be addressed to Kou-Xing Zhang; kxz6210@126.com
}

Received 21 January 2015; Revised 25 March 2015; Accepted 6 April 2015

Academic Editor: Eric N. Villegas

Copyright (C) 2015 Yong-Biao Zhang et al. This is an open access article distributed under the Creative Commons Attribution License, which permits unrestricted use, distribution, and reproduction in any medium, provided the original work is properly cited.

\begin{abstract}
The objective of this study was to estimate the seroprevalence of Toxoplasma gondii infection in 394 patients of intensive care unit (ICU) in a hospital between April 2010 and March 2012 and analyze the association between T. gondii infection and ICU patients according to the species of disease. Toxoplasma serology was evaluated by ELISA method using a commercially available kit. Data of patients were obtained from the patients, informants, and medical examination records. Seventy-four (18.78\%) of 394 patients were positive for anti-T. gondii IgG antibodies demonstrating latent infection. Of these, the highest $T$. gondii seroprevalence was found in the age group of $31-45$ years (27.45\%), and the lowest was found in the age group of $<30$ years (12.5\%). In addition, females $(21.6 \%)$ had a higher seroprevalence than males (18.36\%). With respect to the species of disease, the patients with kidney diseases (57.14\%), lung diseases (27.84\%), and brain diseases (24\%) had high T. gondii seroprevalence. The present study represents the first survey of T. gondii seroprevalence in ICU patients in China, revealing an $18.78 \%$ seropositivity. Considering the particularities of ICU patients, molecular identification, genetic characterization, and diagnosis of $T$. gondii should be considered in future study.
\end{abstract}

\section{Introduction}

Toxoplasmosis, a zoonotic infection of humans and animals, is caused by the ubiquitous obligatory intracellular coccidian protozoan Toxoplasma gondii, which is an opportunistic parasitic infection in immune-compromised hosts worldwide, and approximately one-third of the global population has been computed to be carrying the parasite [1]. Human infection with T. gondii has been reported in China, with a mean infection rate of $7.9 \%$ nationwide, as revealed by ELISA between 2001 and 2004 [2]. However, in recent years, a large amount of epidemiological investigation experiments have been conducted in various groups, including normal population, pregnant women, cancer patients, and psychiatric patients, indicating that $T$. gondii infection is actually a significant human health problem in China [3-6]. T. gondii infections in humans are usually acquired through ingesting tissue cysts of the parasite in raw or undercooked meat, by ingesting parasite oocysts in feline faeces that contaminate drinking water, soil, vegetables, and other food sources, and transplacentally from infected mothers to their infants $[1,7]$.

In immunocompetent individuals, most $T$. gondii infections are asymptomatic. Nevertheless, in immunocompromised hosts, an increased risk of reactivation of latent infection in various organs may occur, resulting in severe diseases or even death [8]. Patients in the ICU are usually considered to be particularly immunocompromised; they may be extremely susceptible to the reactivation of $T$. gondii infection. Thus, detection and surveillance of antiToxoplasma antibodies are of great interest, especially in ICU 
TABLE 1: Seroprevalence of Toxoplasma gondii infection in 394 intensive care unit patients in China.

\begin{tabular}{lccc}
\hline Characteristic & Number of subjects tested & Prevalence (\%) $(95 \%$ CI $)$ & $P$ value \\
\hline Age groups (years) & & & \\
30 or less & 40 & $2.50(2.25-22.75)$ & $27.45(15.20-39.70)$ \\
$31-45$ & 51 & $20.31(10.47-30.17)$ & 0.10 \\
$46-60$ & 64 & $20.00(11.96-28.04)$ & 0.31 \\
$61-75$ & 95 & $17.07(8.93-25.22)$ & 0.30 \\
$>75$ & 82 & $14.52(5.75-23.29)$ & 0.51 \\
No data & 62 & $18.36(13.08-23.63)$ \\
Gender & & $21.60(14.39-28.81)$ & 0.77 \\
Male & 207 & $14.52(5.75-23.29)$ \\
Female & 125 & & 0.47 \\
No data & 62 & & 0.48 \\
\hline
\end{tabular}

TABLE 2: Toxoplasma gondii infection in 394 intensive care unit patients with different species of disease.

\begin{tabular}{lcc}
\hline Species of disease & Number of subjects tested & Prevalence (\%) (95\% CI) \\
\hline Liver disease & 31 & $19.36(5.45-33.26)$ \\
Lung disease & 97 & $27.84(18.92-36.75)$ \\
Kidney disease & 7 & $57.14(20.48-93.80)$ \\
Heart disease & 95 & $11.58(5.15-18.01)$ \\
Brain disease & 50 & $24.00(12.16-35.84)$ \\
Other diseases & 114 & $12.28(6.26-18.31)$ \\
Total & 394 & $18.78(14.93-22.64)$ \\
\hline
\end{tabular}

patients presenting with at least one form of organ failure $[9,10]$. However, epidemiological knowledge regarding the prevalence of T. gondii infection in ICU patients is unavailable in China. Therefore, the present study was conducted to estimate the seroprevalence of $T$. gondii infection in ICU patients in China for the first time, aiming to evaluate the risk for reactivation of $T$. gondii infection in ICU patients in China.

\section{Methods and Materials}

2.1. Ethics Statement. This study was approved before its commencement by the Ethics Committee of the Third Affiliated Hospital, Sun Yat-sen University. The sera were collected with agreement from the volunteers and guardians.

2.2. Serum Samples and Serological Examination. Three hundred and ninety-four (207 male, 125 female, and 62 no data) ICU patients who presented to the Third Affiliated Hospital, Sun Yat-sen University, Guangdong province, China, between April 2010 and March 2012 were included in the study. Each patient required intensive care management, as they displayed at least one form of organ failure. Blood sample was taken from all the ICU patients under sterile conditions, and the sera were separated and stored at $-20^{\circ} \mathrm{C}$ until further testing. In addition, data of patients were obtained from the patients, informants, and medical examination records, including age, gender, and species of disease. All the serum samples were tested for T. gondii IgG antibodies by enzymelinked immunosorbent assay (ELISA) using a commercially available kit (Haitai Co., Ltd., China) according to the manufacturer's instructions. Positive and negative serum controls were included in every plate. All samples were run in triplicate.

2.3. Statistical Analyses. All data were processed and analyzed by SPSS 19.0 Data Editor (SPSS Inc., Chicago, Illinois, USA). $\chi^{2}$-test and Kolmogorov-Smirnov one-sample test were used. All statistical tests were two-sided. The results in comparison with groups were considered different if $P<0.05$.

\section{Results}

Seventy-four (18.78\%, 95\% CI: 14.93\%-22.64\%) of 394 ICU patients were positive for anti-T. gondii IgG antibodies demonstrating latent infection with $T$. gondii. Of these, the highest seroprevalence of T. gondii infection was found in the age group of $31-45$ years $(27.45 \%)$ and the lowest was found in the age group of $<30$ years $(12.50 \%)$. In addition, females $(21.60 \%)$ had a higher seroprevalence than males $(18.36 \%)$, but the difference was not significantly different $(P=0.47)$ (Table 1).

The seroprevalence of $T$. gondii infection in the patients with respect to the species of disease is shown in Table 2, and the highest $T$. gondii seroprevalence was found in patients with kidney diseases (57.14\%), which is significantly higher than others. In addition, high prevalence of latent T. gondii infection was also found in patients with lung diseases (27.84\%, 95\% CI: $18.92 \%-36.75 \%)$ and brain diseases (24.00\%, 95\% CI: $12.16 \%-35.84 \%)$. 


\section{Discussion}

T. gondii infection in humans is common all over the world, with the prevalence varying in accordance with environment, eating habits, and age. In the present study, we found an $18.78 \%$ seroprevalence of T. gondii in ICU patients, which is significantly higher than that in people who lived in Meixian (10.12\%) in Guangdong province [11], and 7.50\% T. gondii seroprevalence in blood donors in Guangdong province [12]. This difference suggests that ICU patients may be more susceptible to T. gondii infection. Therefore, any immunocompromised patients should be requested for the detection of T. gondii infection, and those who are at high risk of being infected should be considered carefully. Moreover, in Guangdong province, wildlife is very popular in their daily diet and it is common to eat dogs and cats for local population. All of these may contribute to the acquisition of T. gondii infection.

Toxoplasmosis was considered to be acquired in the early stage and the prevalence is enhanced with age and declines in later stage $[10,13]$. From the present study, higher seroprevalence of $T$. gondii infection was found in younger population compared to older age group, which was consistent with the abovementioned studies. Toxoplasmosis was considered as an important infectious syndrome, and the main clinical feature is the enlargement of the spleen, the liver, and/or the lymph nodes and it can also influence other organs such as the heart, central nervous system (CNS), or eyes [14]. In the present study, high seroprevalence was found in patients with kidney disease, lung disease, and brain disease, and most of the patients revealed at least one form of organ failure. However, it can not be ignored that the sample size for kidney disease is so small; thus, this warrants further large-scale case-control studies to confirm the present result.

Usually, diagnosis of T. gondii infection can be divided into two categories: direct methods and indirect methods. Direct methods include polymerase chain reaction (PCR), hybridization, isolation, and histology. Indirect methods mainly involve serological methods [15]. In our patients, serology was useful. In immunocompetent patients, indirect serological methods are extensively used because they are faster and cheaper than other methods. However, in asymptomatic patients who are immunocompromised, testing for IgG antibodies to T. gondii should also be executed, as this allows us to identify who are at risk for the reactivation of latent infection [16]. Moreover, detection of IgM antibodies is useful to indicate newly acquired $T$. gondii infection, but the possibility of false IgM positive results should not be discarded.

In immunocompromised patients, direct methods for detecting T. gondii infection must be employed. PCR amplification of T. gondii genes (particularly, the B1 gene) should be employed to detect $T$. gondii in body fluids and tissues of patients [15]. Acute infection could be indicated by directly isolating T. gondii from blood or body fluids, whether newly acquired or reactivation of latent infection. In addition, tissue sections or body fluid smears could also be used to observe tachyzoites.
In conclusion, the present study revealed for the first time the seroprevalence of T. gondii infection in ICU patients in Guangdong province, China. However, some defects are present in the present study: the data represented only a specific province of China and the numbers of patients in the different age groups are small. Further studies should be conducted to estimate the prevalence of T. gondii infection in ICU patients in other provinces of China and increase the size of patients in the different age groups. Second, infection status (current infection or past infection) could not be clearly displayed by the serology, and potential bias could not be excluded due to misclassification. Third, considering association analysis, cross-sectional study design has its shortcoming. Therefore, further large-scale case-control studies or prospective studies should be conducted to confirm our findings.

\section{Conflict of Interests}

All the authors declare no conflict of interests.

\section{Acknowledgment}

This study was supported by the Science Fund for Creative Research Groups of Gansu Province (Grant no. 1210RJIA006).

\section{References}

[1] J. P. Dubey, Toxoplasmosis of Animals and Humans, CRC Press, Boca Raton, Fla, USA, 2nd edition, 2010.

[2] P. Zhou, N. Chen, R.-L. Zhang, R.-Q. Lin, and X.-Q. Zhu, "Foodborne parasitic zoonoses in China: perspective for control," Trends in Parasitology, vol. 24, no. 4, pp. 190-196, 2008.

[3] W. Cong, G.-H. Liu, Q.-F. Meng et al., “Toxoplasma gondii infection in cancer patients: prevalence, risk factors, genotypes and association with clinical diagnosis," Cancer Letters, vol. 359, no. 2, pp. 307-313, 2015.

[4] W. Cong, W. Dong, L. Bai et al., "Seroprevalence and associated risk factors of Toxoplasma gondii infection in psychiatric patients: a case-control study in eastern China," Epidemiology \& Infection, 7 pages, 2015.

[5] Q. F. Meng, H. L. You, N. Zhou, W. L. Dong, W. L. Wang, and W. Cong, "Seroprevalence of Toxoplasma gondii antibodies and associated risk factors among children in Shandong and Jilin provinces, China," International Journal of Infectious Diseases, vol. 30, pp. 33-35, 2015.

[6] X.-J. Gao, Z.-J. Zhao, Z.-H. He et al., “Toxoplasma gondii infection in pregnant women in China," Parasitology, vol. 139, no. 2, pp. 139-147, 2012.

[7] J. G. Montoya and O. Liesenfeld, "Toxoplasmosis," The Lancet, vol. 363, no. 9425, pp. 1965-1976, 2004.

[8] L. Machala, M. Malý, O. Beran, D. Jilich, and P. Kodym, "Incidence and clinical and immunological characteristics of primary Toxoplasma gondii infection in HIV-infected patients," International Journal of Infectious Diseases, vol. 17, no. 10, pp. e892-e896, 2013.

[9] M. Demar, D. Hommel, F. Djossou et al., "Acute toxoplasmoses in immunocompetent patients hospitalized in an intensive care 
unit in French Guiana," Clinical Microbiology and Infection, vol. 18, no. 7, pp. E221-E231, 2012.

[10] A. Nimir, A. Othman, S. Ee et al., "Latent toxoplasmosis in patients with different malignancy: a hospital based study," Journal of Clinical Medicine Research, vol. 2, no. 3, pp. 117-120, 2010.

[11] X. Q. Xie, Y. H. Deng, K. G. Xiao et al., "Study on the Toxoplasma gondii and its control in Meixian," Journal of Tropical Medicine, vol. 4, no. 6, pp. 731-732, 2004.

[12] J. F. Zeng, H. Li, H. Cai, and G. Chen, "Seroepidemiological survey of Toxoplasma gondii in infection in blood donors in Shenzhen City," China Tropical Medicine, vol. 5, no. 3, pp. 599600, 2005.

[13] V. Thomas, B. Sinniah, and P. L. Yap, "Prevalence of antibodies including IgM to Toxoplasma gondii in Malaysians," The Southeast Asian Journal of Tropical Medicine and Public Health, vol. 11, no. 1, pp. 119-125, 1980.

[14] M. Demar, D. Ajzenberg, D. Maubon et al., "Fatal outbreak of human toxoplasmosis along the Maroni River: epidemiological, clinical, and parasitological aspects," Clinical Infectious Diseases, vol. 45, no. 7, pp. e88-e95, 2007.

[15] A. K. Taila, A. S. Hingwe, and L. E. Johnson, "Toxoplasmosis in a patient who was immunocompetent: a case report," Journal of Medical Case Reports, vol. 5, article 16, 2011.

[16] P. Zhou, Z. Chen, H.-L. Li et al., “Toxoplasma gondii infection in humans in China," Parasites \& Vectors, vol. 4, no. 1, article 165, 2011. 


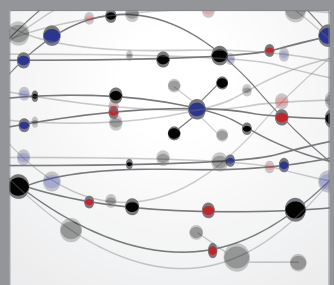

The Scientific World Journal
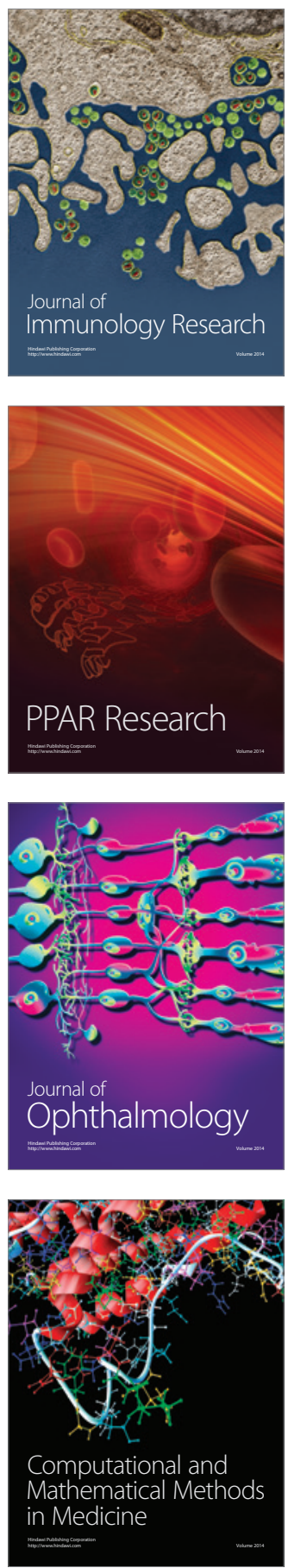

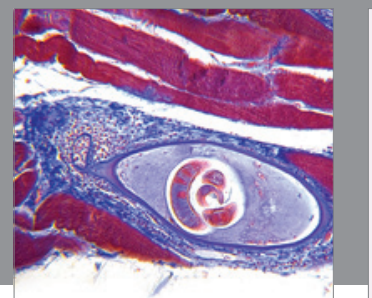

Gastroenterology

Research and Practice
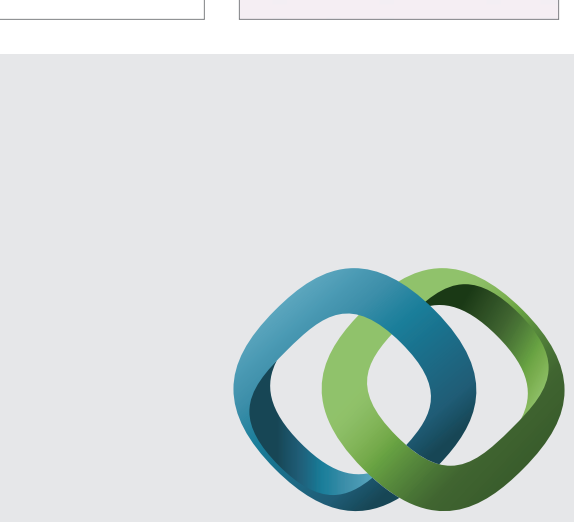

\section{Hindawi}

Submit your manuscripts at

http://www.hindawi.com
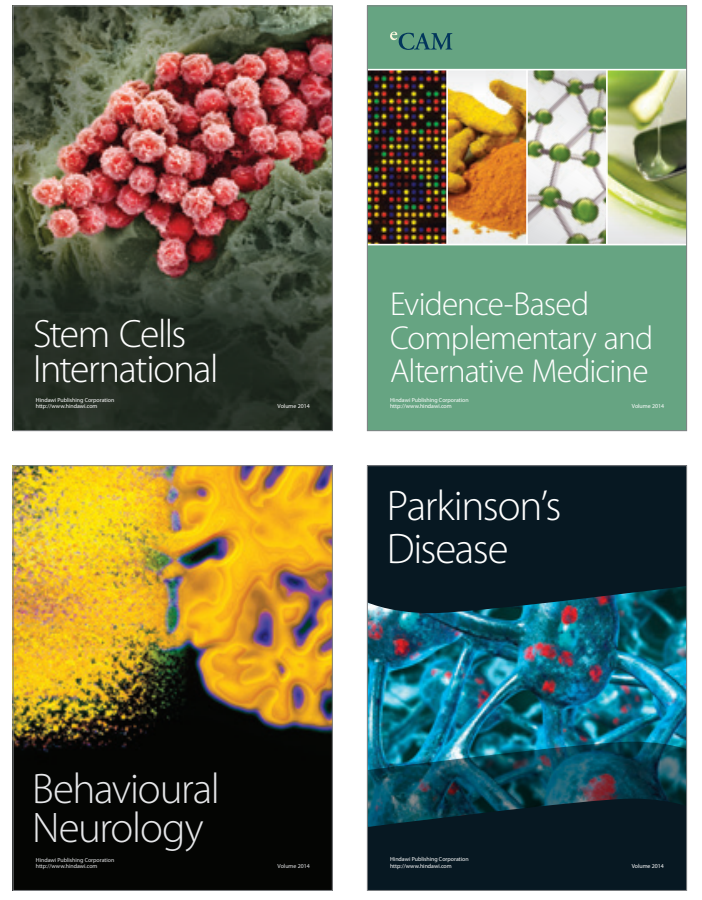
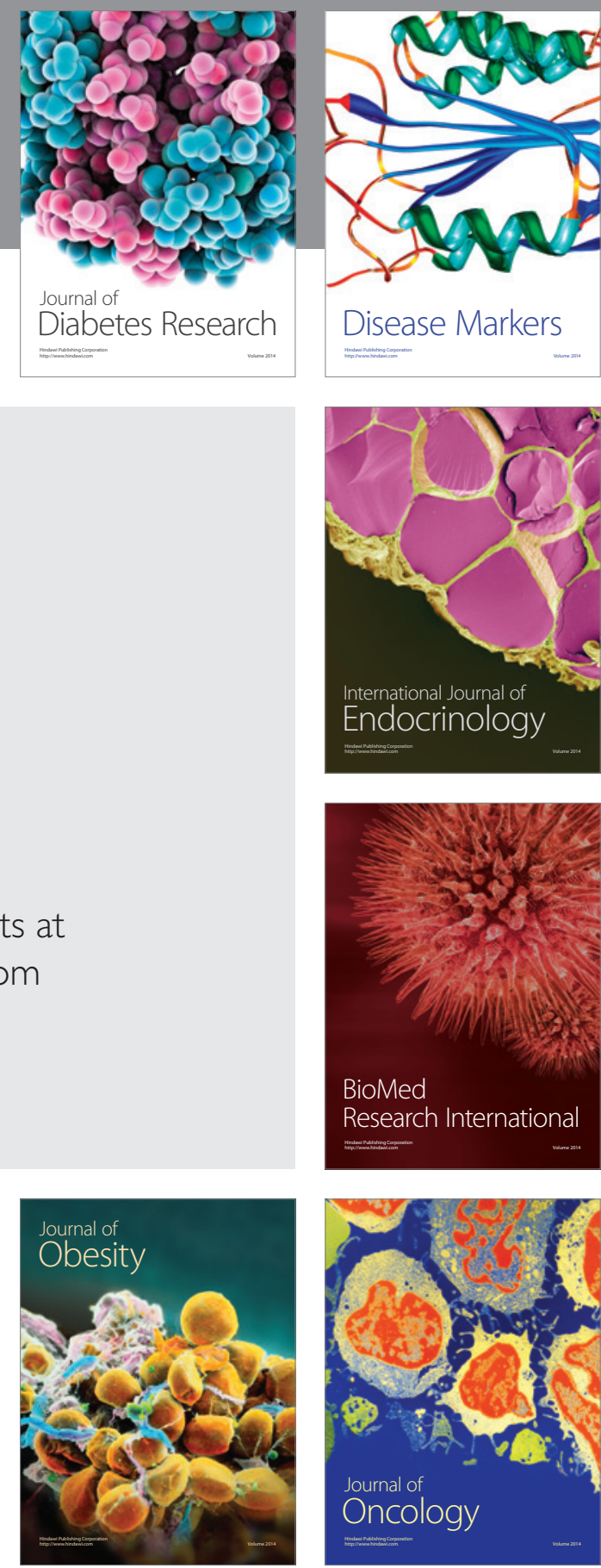

Disease Markers
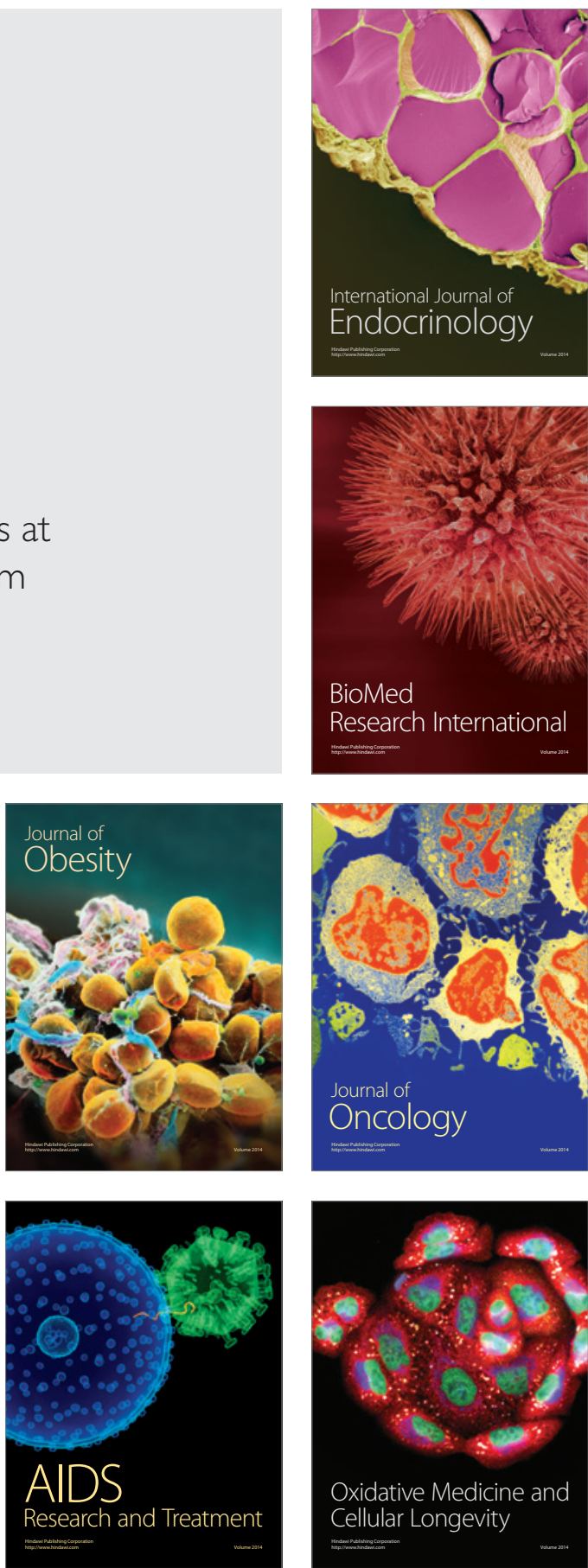